\title{
Radiating-collapsing models satisfying Karmarkar condition
}

\author{
Suresh C. Jaryal ${ }^{\mathrm{a}}$ \\ Department of Physics and Astronomical Science, Central University of Himachal Pradesh (CUHP), Dharamshala, Kangra, HP 176215, India
}

Received: 15 May 2020 / Accepted: 16 July 2020 / Published online: 29 July 2020

(C) The Author(s) 2020

\begin{abstract}
This paper presents a class of exact spherical symmetric solutions of the Einstein equations admitting heatconducting anisotropic fluid as a collapsing matter. The exterior spacetime is assumed to be the Vaidya metric. This class of solutions is shown to satisfy all the energy conditions throughout the interior of the star, and the luminosity is time independent, radiating uniformly throughout the collapse.
\end{abstract}

\section{Introduction}

There has been extensive research in the field of gravitational collapse. Since the pioneering work on the gravitational collapse of homogeneous dust, [1,2], it is now accepted that for a gravitational collapse of homogeneous pressureless matter, the central singularity remains hidden behind the horizon, implying that the end state of the continual gravitational collapse of homogeneous dust cloud must be a black hole [3]. Further studies have examined various aspects of gravitationally collapsing stellar systems for different kinds of matter distributions, and details may be found in $[4-7,11]$. These studies have thrown light to many interesting facts which must hold for the collapse processes to be physically realistic. For example, for the continuous and smooth matching of the interior collapsed spacetime to the exterior Vaidya spacetime over the timelike hypersurface $\Sigma$, the radial pressure must not vanish at the boundary of the collapsing radiant star, but instead be proportional to the heat flux $[13,14]$.

In order to have a physically well behaved model of a gravitation collapse with generic energy momentum tensors, one not only needs to find physically consistent analytical solutions of the Einstein field equations, but also must ensure validity of the energy conditions as well. The practice usually followed are: to specify the spacetime symmetry, or the gravitational potentials, imposing an equation of state, or restricting the matter content to find the solutions of the gravitational

a e-mail: suresh.fifthd@ gmail.com (corresponding author) collapse. However, there exists a class I condition, which is useful to obtain classes of solutions. This condition arise from some well known geometric theorems as follows: First, an $(n+1)$-dimensional space $V^{n+1}$ can be embedded into a pseudo Euclidean space $E^{n+2}$ of dimension $(n+2)$ [15], and that all the spherically symmetric spacetime are in general of class II. Next, the necessary and sufficient condition for any Riemannian space to be embedding class $\mathbf{I}$ is that it satisfies the Karmarkar condition $[16,17]$. Thus the Karmarkar condition is a useful condition which gives new solutions.

Recently, there has been a renewed interest in obtaining solutions using these conditions, [18-20]. The study of the non-static radiating metric with timelike Karmarkar condition, when the temporal dependence of the model is linear, shows that the collapse proceeds without the formation of the horizon [21]. The assumption is that the metric coefficients (which are in general, functions of $r$ and $t$ ), be separable, for example $g_{r r}=b(r) f(t)$ (see the equation (2.1) for example). In their study, they assume one of gravitational potential, which we denote by $b(r)$, to be constant. Next, using the results of the horizon-free collapse model [22] and a linear form of time function $f(t)$ into the Karmarkar condition the other metric function $g_{t t}$ is obtained. It must be noted that this choice of $b(r)$ is special in [21], and one may generalise. We introduce a form of pressure anisotropy $\Delta=p_{t}-p_{r}$, between the radial and the tangential pressures, and using the Karmarkar condition, show that here too the gravitational potentials may be determined. Clearly, these gravitational potentials generalise those in [21]. For these models, we study the thermodynamical evolution and their temperature profiles, since these quantities play prominent role during dissipative gravitational collapse. In fact, they decide the departure from the thermodynamical equilibrium. Quite naturally, the study of these transport processes in the context of irreversible thermodynamics in radiative gravitational collapse has gained considerable attention and [27-35] give further details. 
The paper has been organized as follows. In Sect. 2 we present a description of field equations of spherical symmetric anisotropic system and the junction conditions for smoothly matching of the interior spacetime with the exterior Vaidya spacetime across the timelike surface $\Sigma$. Also, we present the solutions to the Einstein field equation and the explicit expressions for physical quantities. In the light of these solutions we analyze the physical evidences of our model by verifying the energy conditions and it has been shown that all these energy conditions are well satisfied throughout the collapse. In Sect. 3 we study the thermodynamical evolution and temperature profiles of the radiative gravitational collapse. Finally, discussion of the result accompanied with concluding remarks are given in Sect. 4 .

\section{Fluid distribution and field equations}

In comoving-synchronous coordinates, the general spherically symmetric metric is given by

$$
\begin{aligned}
d s^{2}=- & a(r)^{2} d t^{2}+b(r)^{2} f(t)^{2} d r^{2}+r^{2} b(r)^{2} f(t)^{2} \\
& \times\left(d \theta^{2}+\sin ^{2} \theta d \phi^{2}\right) .
\end{aligned}
$$

We consider the interior energy-momentum tensor for the anisotropic fluid distribution with radial heat flow of the form

$T_{\mu \nu}=\left(p_{t}+\rho\right) u_{\mu} u_{\nu}+p_{t} g_{\mu \nu}+\left(p_{r}-p_{t}\right) X_{\mu} X_{\nu}$

$$
+q_{\mu} u_{\nu}+q_{\nu} u_{\mu} \text {, }
$$

where $\rho, p_{r}$ and $p_{t}$ are the energy density, radial pressure and tangential pressure respectively, $u^{\mu}, X^{\mu}$ and $q^{\mu}$ are unit time-like 4-velocity vector, unit space-like vector along radial 4 -vector and radial heat flow vector respectively. These satisfy $u_{\mu} u^{\mu}=-X_{\mu} X^{\mu}=-1$ and $u_{\mu} X^{\mu}=u_{\mu} q^{\mu}=0$. In the comoving co-ordinates the 4 -velocity and unit space-like vector and radial heat flow vector of the fluid are given by

$u^{\mu}=\frac{1}{a} \delta_{0}^{\mu} ; X^{\mu}=\frac{1}{b f} \delta_{1}^{\mu} ; q^{\mu}=\frac{1}{b f} X^{\mu}$.

The magnitude of the expansion scalar $\Theta$ for the metric (2.1) is given by

$\Theta=\nabla \mu u^{v}=\frac{3 \dot{f}}{a f}$.

The non vanishing components of Einstein-Maxwell field equations for the metric (2.1), energy momentum tensor (2.2) and (2.3) are (using units with $c=1=8 \pi G$ )

$$
\begin{aligned}
\rho & =\frac{3 \dot{f}^{2}}{a^{2} f^{2}}-\frac{1}{b^{2} f^{2}}\left(\frac{2 b^{\prime \prime}}{b}-\frac{b^{\prime 2}}{b^{2}}+\frac{4 b^{\prime}}{r} \frac{b}{b}\right), \\
p_{r} & =-\frac{1}{a^{2}}\left(\frac{2 \ddot{f}}{f}+\frac{\dot{f}^{2}}{f^{2}}\right)+\frac{1}{b^{2} f^{2}}
\end{aligned}
$$

$$
\begin{aligned}
& \times\left(\frac{2 a^{\prime} b^{\prime}}{a b}+\frac{2}{r}\left(\frac{a^{\prime}}{a}+\frac{b^{\prime}}{b}\right)+\frac{b^{\prime 2}}{b^{2}}\right), \\
p_{t}=- & \frac{1}{a^{2}}\left(\frac{2 \ddot{f}}{f}+\frac{\dot{f}^{2}}{f^{2}}\right)+\frac{1}{b^{2} f^{2}} \\
& \times\left(\frac{a^{\prime \prime}}{a}+\frac{1}{r}\left(\frac{a^{\prime}}{a}+\frac{b^{\prime}}{b}\right)-\frac{b^{\prime 2}}{b^{2}}+\frac{b^{\prime \prime}}{b}\right), \\
q=- & \frac{2 a^{\prime} \dot{f}}{a^{2} b^{2} f^{3}} .
\end{aligned}
$$

where dot and prime are the derivatives with respect to $t$ and $r$ respectively. From Eqs. (2.5)-(2.8) we can see that the number of field equations are less than that of the number of unknowns. Also, the form of three metric potentials fix all the unknown physical quantities of the system. So, in order to study the collapsing phenomena we need to fix forms for these metric potentials.

Let us begin with the Israel-Darmois junction conditions [36-38]. The interior manifold is $\mathbf{M}^{-}$and exterior manifold is $\mathbf{M}^{+}$to be matched across the bounding timelike three space $\Sigma$, at $r=r_{b}$. The exterior spacetime $\mathbf{M}^{+}$, is described by the Vaidya spacetime having outgoing radial flow of the radiation given by [12]

$$
\begin{aligned}
d s_{+}^{2}= & -\left(1-\frac{2 M(v)}{\mathbf{r}}\right) d v-2 d v d \mathbf{r} \\
& +\mathbf{r}^{2}\left(d \theta^{2}+\sin ^{2} \theta d \phi^{2}\right),
\end{aligned}
$$

The junction conditions require the matching of metric as well as the extrinsic curvatures

$$
\begin{aligned}
d s_{\Sigma}^{2} & =\left(d s_{-}\right)_{\Sigma}^{2}=\left(d s_{+}\right)_{\Sigma}^{2} \\
{\left[K_{i j}\right]_{\Sigma} } & =K_{i j}^{+}=K_{i j}^{-},
\end{aligned}
$$

where $K_{i j}^{ \pm}$is the extrinsic curvature to $\Sigma$ given by [42]

$K_{i j}^{ \pm}=-n_{l}^{ \pm} \frac{\partial^{2} x^{l}}{d z^{i} d z^{j}}-n_{l}^{ \pm} \Gamma_{m n}^{l} \frac{\partial x^{m}}{d z^{i}} \frac{\partial x^{n}}{d z^{j}}$.

Here $x^{l}$ are the coordinates of interior and exterior spacetimes, $z^{i}$ are the coordinates that defines the hypersurface $\Sigma$ and $n^{i}$ are the unit normal vector to $\Sigma$.

The junction condition on metric functions, given by (2.10) at the hypersurface $\Sigma$ gives

$$
\begin{aligned}
d t & =a(r)_{\Sigma}^{-1} d \tau, \\
\mathbf{r}_{\Sigma}(v) & =(r b f)_{\Sigma}, \\
\left(\frac{d v}{d \tau}\right)_{\Sigma}^{-2} & =\left(1-\frac{2 M}{\mathbf{r}}+2 \frac{d \mathbf{r}}{d v}\right)_{\Sigma},
\end{aligned}
$$

where $\tau$ is the time coordinate defined only on the hypersurface $\Sigma$. To match the extrinsic curvatures, we need the normal vector fields. The unit normal vectors on the hypersurface $\Sigma$ for the interior and exterior spacetime are given 
by

$n_{l}^{-}=\left[0,(b f)_{\Sigma}, 0,0\right]$,

$n_{l}^{+}=\left(1-\frac{2 M}{\mathbf{r}}+2 \frac{d \mathbf{r}}{d v}\right)_{\Sigma}^{-\frac{1}{2}}\left(-\frac{d \mathbf{r}}{d v} \delta_{l}^{0}+\delta_{l}^{1}\right)_{\Sigma}$.

The non vanishing components of the extrinsic curvature for metrics (2.1) and (2.9) are given by

$K_{\tau \tau}^{-}=-\left[\frac{a^{\prime}}{a b f}\right]_{\Sigma}$

$K_{\theta \theta}^{-}=\left[r b f\left(1+\frac{r b^{\prime}}{b}\right)\right]_{\Sigma}$,

$K_{\tau \tau}^{+}=\left[\frac{d^{2} v}{d \tau^{2}}\left(\frac{d v}{d \tau}\right)^{-1}-\left(\frac{d v}{d \tau}\right) \frac{M}{\mathbf{r}^{2}}\right]_{\Sigma}$,

$K_{\theta \theta}^{+}=\left[\left(\frac{d v}{d \tau}\right)\left(1-\frac{2 M}{\mathbf{r}}\right) \mathbf{r}-\mathbf{r} \frac{d \mathbf{r}}{d \tau}\right]_{\Sigma}$,

$K_{\phi \phi}^{-}=\sin ^{2} \theta K_{\theta \theta}^{-}, K_{\phi \phi}^{+}=\sin ^{2} \theta K_{\theta \theta}^{+}$.

Now, from the second junction condition (2.11), one must have the equality for the $\theta \theta$ components since the spherical part matches for the interior and the exterior. Thus, the equation $K_{\theta \theta}^{+}=K_{\theta \theta}^{-}$at hypersurface $\Sigma$, and along with Eqs. (2.13), (2.14) and (2.15) give

$\left[r b f\left(1+\frac{r b^{\prime}}{b}\right)\right]_{\Sigma}=\left[\left(\frac{d v}{d \tau}\right)\left(1-\frac{2 M}{\mathbf{r}}+\frac{Q^{2}}{\mathbf{r}^{2}}\right) \mathbf{r}-\mathbf{r} \frac{d \mathbf{r}}{d \tau}\right]_{\Sigma}$,

$m_{\Sigma}=\left[\frac{r^{3} \dot{f}^{2} b^{3} f}{2 a^{2}}-\frac{r^{3} f b^{\prime 2}}{2 b}-r^{2} f b^{\prime}+\frac{Q^{2}}{2 r b f}\right]_{\Sigma}$,

where $2 m$ is the total energy entrapped inside the hypersurface $\Sigma[40,41]$. Now, again from the matching condition (2.11), the matching of the $K_{\tau \tau}^{+}=K_{\tau \tau}^{-}$component together with the Eq. (2.13) we have

$$
-\left[\frac{a^{\prime}}{a b f}\right]_{\Sigma}=\left[\frac{d^{2} v}{d \tau^{2}}\left(\frac{d v}{d \tau}\right)^{-1}-\left(\frac{d v}{d \tau}\right) \frac{M}{\mathbf{r}^{2}}\right]_{\Sigma} .
$$

Substituting Eqs. (2.13), (2.14) and (2.23) into the Eq. (2.22) we have

$$
\left(\frac{d v}{d \tau}\right)_{\Sigma}=\left(1+\frac{r b^{\prime}}{b}+\frac{r b \dot{f}}{a}\right)_{\Sigma}^{-1} .
$$

Now, differentiating (2.25) with respect to the $\tau$ and using Eqs. (2.23) and (2.25), we can write the Eq. (2.24) and comparing with Eqs. (2.6) and (2.8) we have the useful equation

$\left(p_{r}\right)_{\Sigma}=(q b f)_{\Sigma}$
We also require the total Luminosity for an observer at rest at infinity is given by $[9,10]$

$L_{\infty}=-\left(\frac{d m}{d v}\right)_{\Sigma}=-\left[\frac{d m}{d t} \frac{d t}{d \tau}\left(\frac{d v}{d \tau}\right)^{-1}\right]_{\Sigma}$.

Differentiating the Eq. (2.23) with respect to $t$ and using Eqs. (2.6), (2.13) and (2.24), above Eq. (2.27) becomes

$L_{\infty}=\left[\frac{r^{2} f^{2} b^{2} p_{r}}{2}\left(1+\frac{r b^{\prime}}{b}+\frac{r b \dot{f}}{a}\right)^{2}\right]_{\Sigma}$.

The boundary redshift can be used to determine the time of formation of the horizon. The boundary redshift $Z_{\Sigma}$ is given by $[9,10]$

$\left(\frac{d v}{d \tau}\right)_{\Sigma}=1+Z_{\Sigma}$

As usual, with $\left(1+\frac{r b^{\prime}}{b}+\frac{r b \dot{f}}{a}\right)_{\Sigma}=0$, for an observer at rest at infinity, the red shift diverges at the time of formation of the blackhole. Now, to find the solutions of the field Eqs. (2.5)-(2.8), one need to have the form of the metric potential. From Eqs. (2.6), (2.7) and (2.1), the pressure anisotropy factor $\Delta=p_{t}-p_{r}$ has the form

$\Delta=\frac{1}{f^{2} b^{2}}\left[\frac{a^{\prime \prime}}{a}+\frac{b^{\prime \prime}}{b}-\frac{1}{r}\left(\frac{a^{\prime}}{a}+\frac{b^{\prime}}{b}\right)-\frac{2 a^{\prime} b^{\prime}}{a b}-\frac{2 b^{\prime 2}}{b^{2}}\right]$.

Since the number of field equations are more than that of the number of unknowns in the system. These unknowns depend upon the form of metric potentials. To find the metric potentials, we take the pressure anisotropy $\Delta$ to be:

$\Delta=\frac{1}{f^{2} b^{2}}\left[\frac{a^{\prime \prime}}{a}-\frac{a^{\prime}}{r a}-\frac{2 a^{\prime} b^{\prime}}{a b}\right]$.

The full expression of anisotropy is given in Eq. (2.30). However, this form is too complicated to be solved in full detail. So, to simplify, we separate a set of terms which are significant in the following sense: the anisotopy in pressure $\Delta$ is so assumed that the model is physically significant, such that $\Delta$ vanishes at the center $r=0$ of the cloud and is regular towards the boundary. Furthermore, this choice makes the original pressure anisotropic Eq. (2.30) as differential equation of only one function, which one can easily integrate to find solution. The vanishing part of the Eq. (2.30)

$0=\frac{1}{f^{2} b^{2}}\left[\frac{b^{\prime \prime}}{b}-\frac{b^{\prime}}{r b}-\frac{2 b^{\prime 2}}{b^{2}}\right]$,

which gives the following solution for the function $b(r)$ :

$b(r)=-\frac{2}{C_{3} r^{2}+2 C_{4}}$, 
where $C_{3}$ and $C_{4}$ are constant of integration.

One may now obtain time dependent solutions. It is known that an $(n+1)$-dimensional space $V^{n+1}$ can be embedded into a pseudo Euclidean space $E^{n+2}$ of dimension $(n+2)$ if there exists a symmetric tensor $b_{\mu \nu}$ which satisfies the Gauss-Codazzi equations [15].

$$
\begin{aligned}
R_{\mu \nu \lambda \delta} & =2 e b_{\mu[\lambda} b_{\delta] \nu}, \\
0 & =b_{\mu[v ; \lambda]}-\Gamma_{[\nu \lambda]}^{\sigma} b_{\mu \sigma}+\Gamma_{\mu[v}^{\sigma} b_{\lambda] \sigma},
\end{aligned}
$$

where $e= \pm 1$ ( + or - , when the normal to the manifold is spacelike or timelike respectively) and $b_{\mu \nu}$ are the coefficient of the second differential form. The necessary and sufficient condition for any Riemannian space to be an embedding class I is the Karmarkar condition [16,17], and for our case, the condition reduces to:

$R_{\text {rtrt }} R_{\theta \phi \theta \phi}=R_{r \theta r \theta} R_{t \phi t \phi}-R_{\theta r t \theta} R_{\phi r t \phi}$.

The non vanishing components of the Riemann tensor for the shear free spherically symmetric metric (2.1) are

$$
\begin{aligned}
R_{r t r t} & =a^{2}\left(\frac{a^{\prime \prime}}{a}-\frac{b^{2} f}{a^{2}} \ddot{f}-\frac{a^{\prime}}{a} \frac{b^{\prime}}{b}\right), \\
R_{\theta \phi \theta \phi} & =r^{4} b^{2} f^{2}\left(\frac{b^{2}}{a^{2}} \dot{f}^{2}-\frac{2 b^{\prime}}{r b}-\frac{b^{\prime 2}}{b^{2}}\right) \sin ^{2} \theta, \\
R_{r \theta r \theta} & =r^{2} b^{2} f^{2}\left(\frac{b^{2}}{a^{2}} \dot{f}^{2}-\frac{b^{\prime}}{r b}-\frac{b^{\prime \prime}}{b}+\frac{b^{\prime 2}}{b^{2}}\right), \\
R_{t \phi t \phi} & =r^{2} a^{2} b\left(\frac{a^{\prime}}{r a}-\frac{b^{2} f}{a^{2}} \ddot{f}+\frac{a^{\prime}}{a} \frac{b^{\prime}}{b}\right) \sin ^{2} \theta, \\
R_{\theta r t \theta} & =\frac{r^{2} b^{2} f}{a} a^{\prime} \dot{f}, \\
R_{\phi r t \phi} & =\sin ^{2} \theta R_{\theta r t \theta} .
\end{aligned}
$$

Using Eqs. (2.37)-(2.42) into the Eq. (2.36) we have the class I condition as

$$
\begin{aligned}
0= & b^{2} \dot{f}^{2} b^{3}\left(\frac{a^{\prime \prime}}{a}-\frac{a^{\prime}}{r a}+\frac{a^{\prime 2}}{a^{2}}-2 \frac{a^{\prime}}{a} \frac{b^{\prime}}{b}\right) \\
& +r^{2} b^{3} f \ddot{f}\left(\frac{b^{\prime}}{r b}+2 \frac{b^{\prime 2}}{b^{2}}-\frac{b^{\prime \prime}}{b}\right) \\
& +r^{2} a a^{\prime} b^{\prime \prime}\left(\frac{1}{r}+\frac{b^{\prime}}{b}\right)-r^{2} a b a^{\prime \prime}\left(2 \frac{b^{\prime}}{r b}+\frac{b^{\prime 2}}{b^{2}}\right) \\
& +r a b a^{\prime}\left(2 \frac{b^{\prime 2}}{b^{2}}+\frac{b^{\prime}}{r b}\right) .
\end{aligned}
$$

Although we have found the form of one of the metric potential $b(r)$ from the choice of anisotropy factor, however, the class I condition Eq. (2.43) is still nonlinear in its temporal and radial behavior. For a collapsing model both the conditions (2.26) and (2.43) must be simultaneously satisfied. It has been found that one of the solutions of (2.43) is a linear solution [22]

$f(t)=-C_{Z} t$

where $C_{Z}>0$. Using Eqs. (2.33) and (2.44) into the above equation (2.43) we obtain the form of $a(r)$ as

$a(r)=\frac{1}{2 \sqrt{2} \sqrt{C_{3} C_{4}}} \sqrt{C_{4}^{2}\left(C_{1} b(r)+4 C_{2} C_{3}\right)^{2}-4 C_{Z}^{2}}$

where $C_{1}$ and $C_{2}$ are integration constants.

The dependency of gravitational potentials $a(r)$ and $b(r) f(t)$ in perfect fluid and the Karmarkar condition leads to two exact solutions: the Schwarzschild [23] or the Kohler and Chao [24] solution. The Kohler and Chao solution is only physical for unbound configuration such as cosmological model as the radial pressure vanishes at $r \rightarrow \infty$. The pressure isotropy condition $\Delta=0$ and the Karmarkar condition (2.43) are satisfied when either $C_{1}, C_{3}$ or $C_{4}$ vanish. However, for the metric potential $a(r)$ to be positive and greater than zero, both $C_{3}$ and $C_{4}$ should not vanish throughout the collapse. This implies that in the static case, when $f(t)=1$, from pressure isotropy and the Karmarkar condition we must have $C_{1}=0$. Thus, the for static case, Karmarkar condition together with the pressure isotropy yields the Schwarzschild like form of the gravitational potentials given by

$a(r)^{2}=\frac{4 C_{2}^{2} C_{3}^{2} C_{4}^{2}-1}{2 C_{3} C_{4}}$,

$b(r)^{2}=\frac{4}{\left(2 C_{4}+C_{3} r^{2}\right)^{2}}$.

Now physical quantities (2.5)-(2.8) interms of these exact solutions of the radiating star becomes

$$
\begin{aligned}
\rho= & \frac{6 C_{3} C_{4}^{3}}{S_{1} C_{Z}^{2} t^{2}}\left(C_{1}-2 C_{2} C_{3}\left(2 C_{4}+C_{3} r^{2}\right)\right)^{2} \\
p_{r}= & \frac{C_{3} C_{4}^{2}}{S_{1} C_{Z}^{2} t^{2}}\left[2 C_{1} C_{2} C_{3}\left(12 C_{4}^{2}+4 C_{3} C_{4} r^{2}-C_{3} r^{2}\right)\right. \\
& \left.-C_{1}^{2}\left(4 C_{4}-C_{3} r^{2}\right)-8 C_{2}^{2} C_{3}^{2} C_{4}\left(2 C_{4}+C_{3} r^{2}\right)^{2}\right] \\
p_{t}= & \frac{C_{3} C_{4}^{2}}{S_{1} C_{Z}^{2} t^{2}}\left[C_{1}^{4} C_{4}^{2}\left(C_{3} r^{2}-4 C_{4}\right)+2 C_{1}^{3} C_{2} C_{3} C_{4}^{2}\right. \\
& \left(-3 C_{3}^{2} r^{4}+8 C_{3} C_{4} r^{2}+28 C_{4}^{2}\right) \\
& +2 C_{1}^{2}\left(C_{3} r^{2}+2 C_{4}\right)^{2}\left(6 C_{2}^{2} C_{3}^{2} C_{4}^{2}\left(C_{3} r^{2}-6 C_{4}\right)\right. \\
& \left.+C_{Z}^{2}\left(2 C_{4}-C_{3} r^{2}\right)\right) \\
& +2 C_{1} C_{2} C_{3}\left(C_{3} r^{2}+2 C_{4}\right)^{3}\left(C_{Z}^{2}\left(C_{3} r^{2}-6 C_{4}\right)\right. \\
& \left.-4 C_{2}^{2} C_{3}^{2} C_{4}^{2}\left(C_{3} r^{2}-10 C_{4}\right)\right)
\end{aligned}
$$




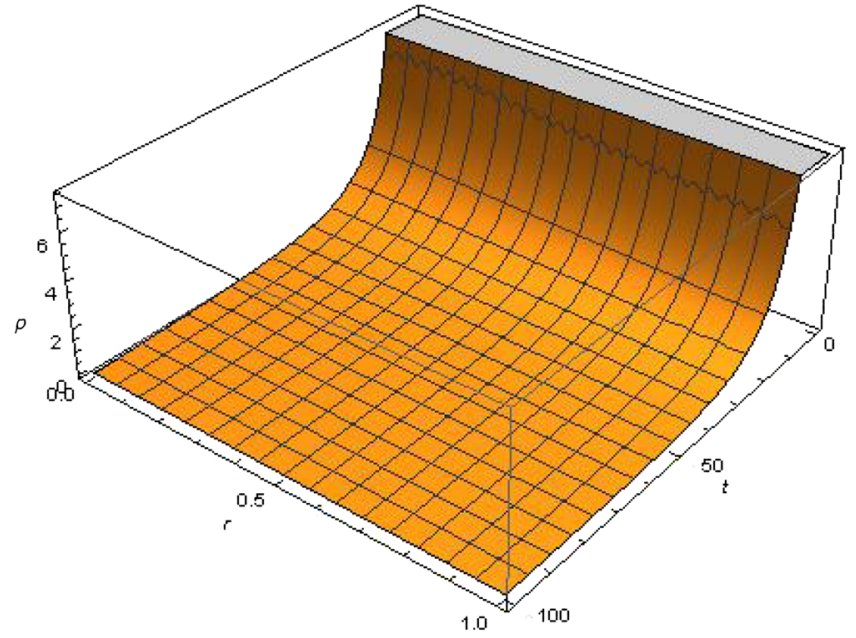

(a)

Fig. 1 a Plot of the density $\rho$, Eq. (2.48), with respect to $r$ at center $r=0$ and at the surface of the star at $r=1$. This plot shows that the density is positive as the collapse starts at $t=-100$ and is maximum at the end state of the collapse at $t=0$. b Plot of the radial pressure

$$
\begin{array}{r}
\left.-8 C_{2}^{2} C_{3}^{2} C_{4}\left(C_{3} r^{2}+2 C_{4}\right)^{4}\left(4 C_{2}^{2} C_{3}^{2} C_{4}^{2}-C_{Z}^{2}\right)\right], \quad(2.50) \\
q=-\frac{r C_{1} C_{4}^{5 / 2} C_{3}^{3 / 2}}{C_{Z}^{2} t^{3}} \frac{4 \sqrt{2}\left[4 C_{2} C_{3}-\frac{2 C_{1}}{C_{3} r^{2}+2 C_{4}}\right]}{\left[C_{4}^{2}\left(4 C_{2} C_{3}-\frac{2 C_{1}}{C_{3} r^{2}+2 C_{4}}\right)^{2}-4 C_{Z}^{2}\right]^{3 / 2}},
\end{array}
$$

The boundary condition (2.26) in the view of (2.49)-(2.51) becomes

$$
2 f \ddot{f}+\dot{f}^{2}-2 x \dot{f}=y,
$$

where

$$
\begin{aligned}
& x=\left(\frac{a^{\prime}}{b}\right)_{\Sigma}, \\
& y=\left(\frac{a^{2}}{b^{2}}\left[\frac{b^{\prime 2}}{b^{2}}+\frac{2}{r}\left(\frac{b^{\prime}}{b}+\frac{a^{\prime}}{a}\right)+\frac{2 a^{\prime} b^{\prime}}{a b}\right]\right)_{\Sigma} .
\end{aligned}
$$

It can be seen from the Figs. 1a, b and 2 a that density, radial pressure and tangential pressure are positive throughout the collapse. Also, Fig. 2b shows that the radial heat flux is also positive throughout collapse. Also the expansion scalar (2.4) and the Misner sharp mass function (2.23) have the form

$$
\begin{aligned}
& \Theta=\frac{6 \sqrt{C_{3} C_{4}}}{t \sqrt{C_{4}^{2}\left(2 C_{2} C_{3}-\frac{C_{1}}{C_{3} r^{2}+2 C_{4}}\right)-2 C_{Z}^{2}}}, \\
& m=\frac{8 t r^{3} C_{3} C_{4}^{3} C_{Z}}{\left(C_{3} r^{2}+2 C_{4}\right)^{3}}
\end{aligned}
$$

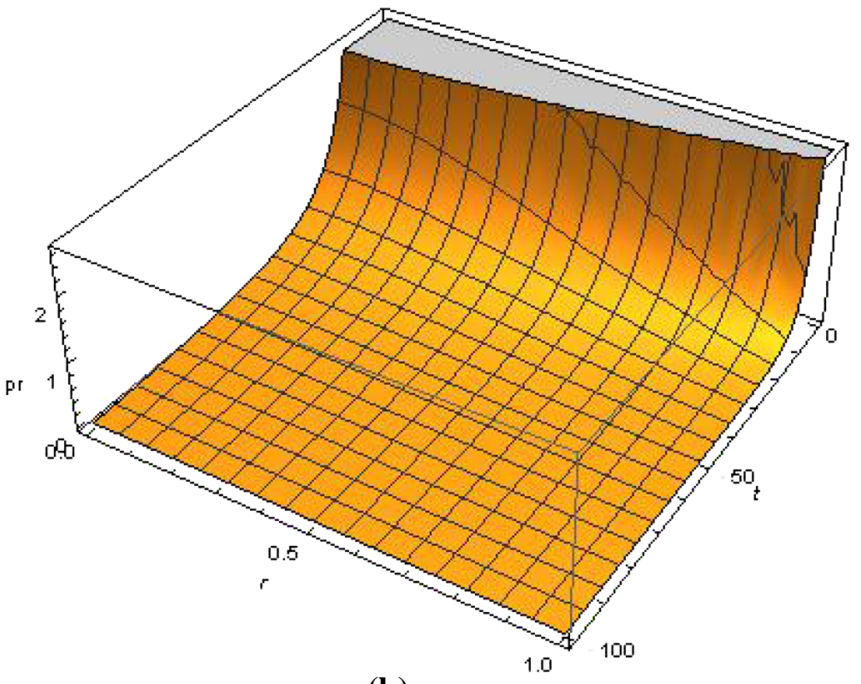

(b)

$p_{r}$, Eq. (2.49), with respect to $r$ at center $r=0$ and at the surface of the star at $r=1$. This plot shows that the radial pressure is positive as the collapse starts at $t=-100$ and is maximum at the end state of the collapse at $t=0$

$$
\times\left[\frac{2 C_{2} C_{3}\left(C_{3} r^{2}+2 C_{4}\right)-C_{1}}{2\left(C_{3} r^{2}+2 C_{4}\right)\left(C_{2} C_{3} C_{4}^{2}-C_{Z}^{2}\right)-C_{1} C_{4}^{2}}\right] .
$$

The metric potentials $a(r)$ and $b(r)$ should be greater than zero throughout the collapsing phenomena. This implies for $b(r)>0$, both $C_{3}$ and $C_{4}$ should be negative. For second metric potential to be positive i.e. $a(r)>0$ we must have $C_{Z}^{2}<C_{4}^{2}\left[\frac{C_{1}}{C_{3} r^{2}+2 C_{4}}-2 C_{2} C_{3}\right]^{2}$. This implies at the center $r=0, C_{Z}<C_{1}-2 C_{2} C_{3} C_{4}$. Also, the condition of positivity of the radial pressure $p_{r}$ throughout the collapsing phenomena imposes constraints on the range of $C_{1}$ as $C_{2} C_{3} C_{4}<C_{1}<2 C_{2} C_{3} C_{4}$.

Using Eqs. (2.33), (2.44), (2.45) and (2.49) into the (2.28), we found that the Luminosity of the radiating collapse becomes time independent and vanishes if $R^{\prime}=\left(b+r b^{\prime}\right)=$ 0 . Which implies that the red shift diverges at the time of formation of the blackhole i.e. at $R^{\prime}=0$. As we know that $R^{\prime}$ implies the shell crossing singularity and for a smooth collapse there should be no shell crossing during the collapse, which means shell crossing singularity should occur either at the same or later epoch than that of shell focusing singularity( i.e at $R=0$ ). As the red shift diverges at $R^{\prime}=0$, this means the only place where blackhole can form is at the central singularity where both the shells crossing and shell focusing singularity forms simultaneously. This is because for a physically realistic smooth model there should be $R^{\prime}>0$ throughout the collapse. It is clear from the Fig. 3a that the expansion scalar $\Theta$ is negative throughout implying 


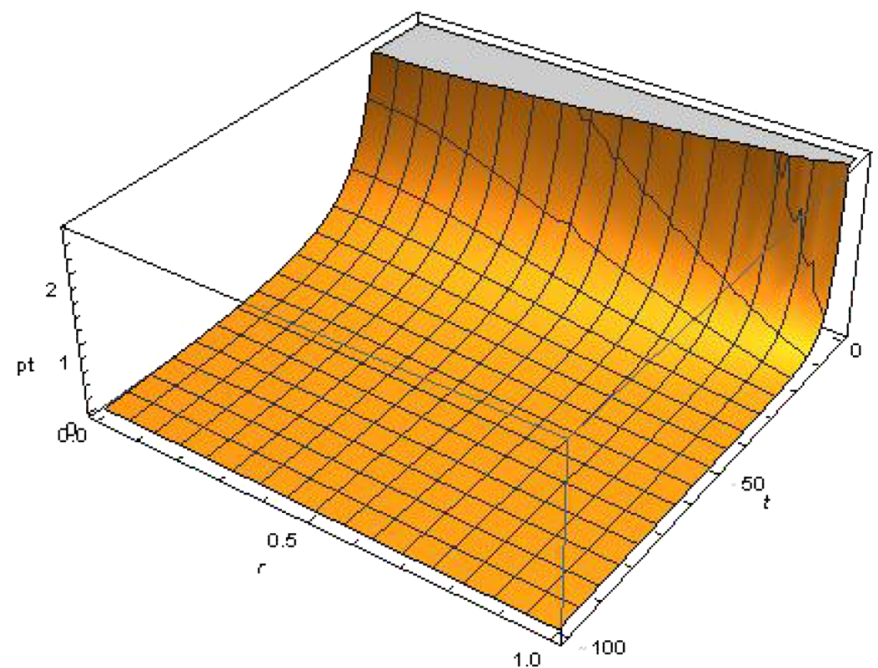

(a)

Fig. 2 a Plot of the tangential pressure $p_{t}$, Eq. (2.50), with respect to $r$ at center $r=0$ and at the surface of the star at $r=1$. This plot shows that the tangential pressure is also positive as the collapse starts at $t=-100$ and is maximum at the end state of the collapse at $t=0$. $\mathbf{b}$ Plot of the radial heat flux $q$, Eq. (2.51), with respect to $t$ and $r$. This plot shows that the heat flux is positive, implying heat is radiating through-

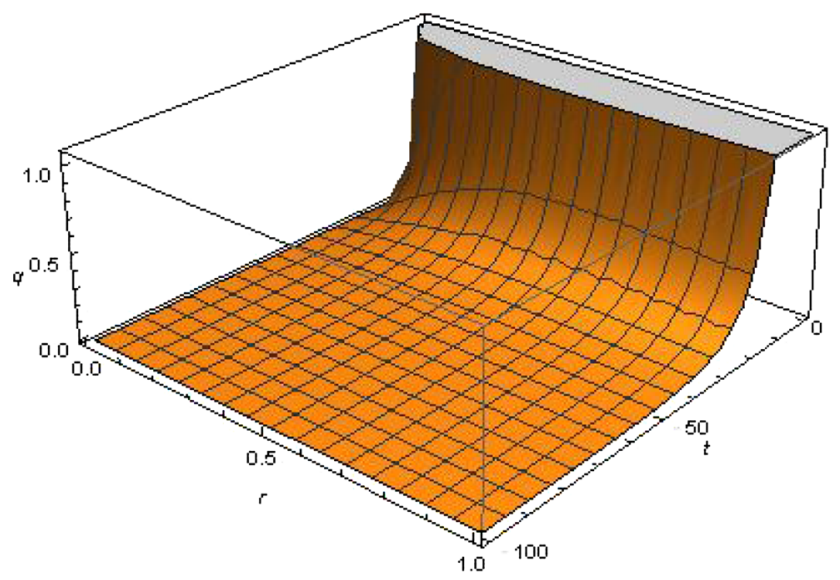

(b)

out the collapse. As the star deviates from the equilibrium, it will start radiating heat and as the contraction of the cloud increases it will start radiating more and more heat flux. It can be seen from the graph that the heat flux starts to increase as the collapse starts at $t=-100$ and heat flux is positive and maximum as it reaches the end state of the collapse at $t=0$

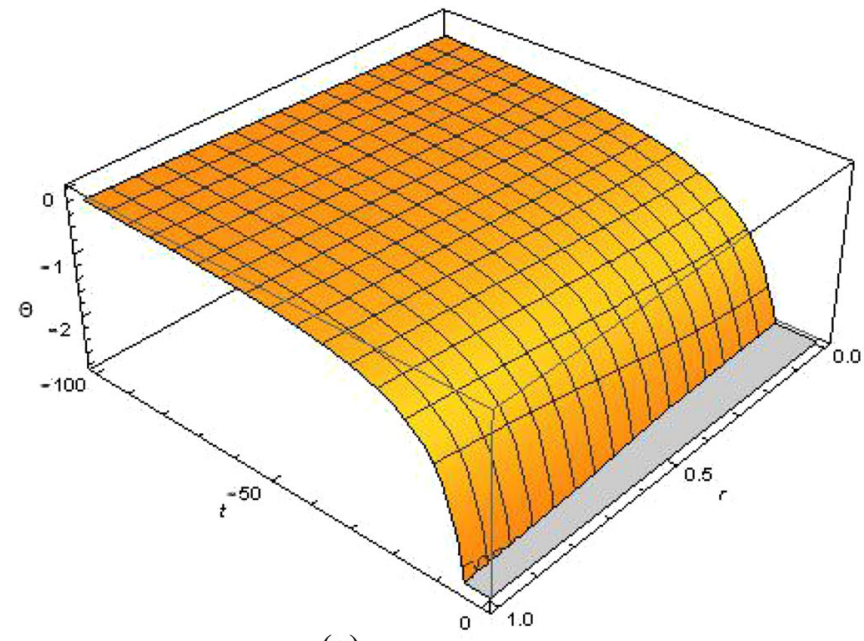

(a)

Fig. 3 a Plot of the expansion scalar $\Theta$, equation (2.55), with respect to $t$ and $r$. For the collapsing phenomena $\Theta$ should be negative throughout the collapse which is confirmed from the figure that as the collapse starts at $t=-100, \Theta$ has zero value and it starts decreasing till the

the contracting behavior of the system. Also, Fig. 3b shows that the mass is linear function of time $t$.

Recently, the Karmarkar scalar condition has been used for the nonstatic system, where they found two class of solutions [26]. One of their solutions is that of a horizon free radiating

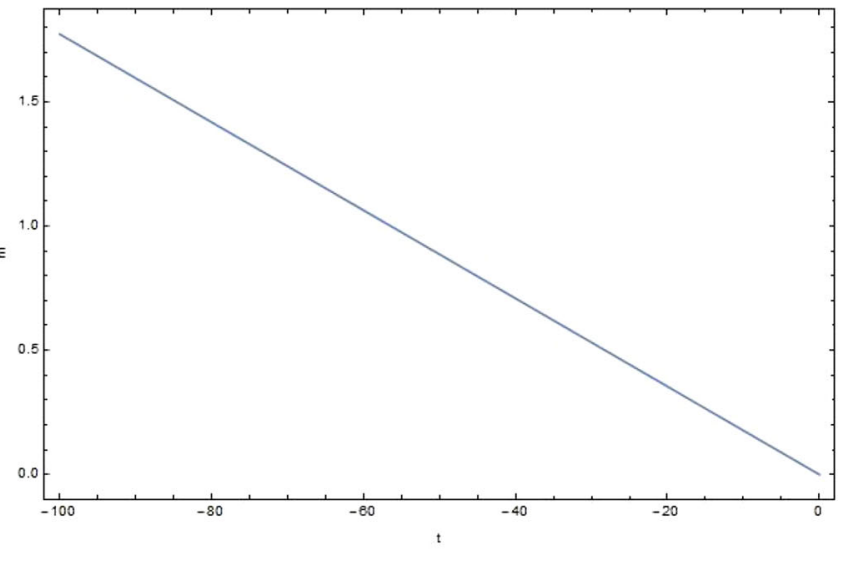

(b)

collapse reaches its end state at $t=0$. b Plot of the mass $m$, Eq. (2.56) of the star with respect to $t$. It can be seen that the mass is linear function of time coordinate and the mass radiates throughout the collapse

collapse as in [21]. However, the second class of solutions are

$A(t, r)=-\dot{\bar{b}}(t) \frac{\sqrt{C(-2+\bar{a} C)+\bar{a} r^{4}\left(-1+\bar{a}^{2} C^{2}\right)+2 r^{2}\left(-1-\bar{a} C+\bar{a}^{2} C^{2}\right)}}{\sqrt{2}\left(1+\bar{a} r^{2}\right)}$,

$B(t, r)=\frac{\bar{b}(t)}{\left(1+\bar{a} r^{2}\right)} \quad ; \quad R(t, r)=r B(t, r)$, 
It must be noted that, for $C_{4}=1, C_{Z}=1, C_{1}=$ $2 C_{Z}, \bar{b}(t)=f(t)=-C_{Z} t, \bar{a}=C_{3} / 2, C(t)=-C_{2}$ the (2.57)-(2.58) reduce to those obtained here using anisotropy together with the Karmarkar condition. Thus, although we had chosen $\Delta$ for the mathematical simplicity, the results obtained are physically significant and in agreement with those obtained in [26].

Let us now ask if the solutions also satisfy the energy conditions as well. Energy conditions plays important role in the study of the astronomical phenomena like collapsing stellar models. In this section we will analyze the physical evidences of our model by verifying the energy conditions. The energy conditions namely weak energy condition(WEC), null energy condition (NEC), dominant energy condition (DEC) and strong energy conditions (SEC) will be satisfied at all points in the stellar model if the following inequalities are satisfied simultaneously $[9,10,23]$
E1: $\left(\rho+p_{r}\right)^{2}-4 q^{2} \geq 0$
(SEC/
$\mathrm{DEC} / \mathrm{WEC})$
E2: $\rho-p_{r} \geq 0$
E3: $\rho-p_{r}-2 p_{t}+\sqrt{\left(\rho+p_{r}\right)^{2}-4 q^{2}} \geq 0$
E4: $\rho-p_{r}+\sqrt{\left(\rho+p_{r}\right)^{2}-4 q^{2}} \geq 0$ (DEC/WEC)
E5: $\rho-p_{r}+2 p_{t}+\sqrt{\left(\rho+p_{r}\right)^{2}-4 q^{2}} \geq 0 \quad$ (SEC/DEC/ WEC)
E6: $2 p_{t}+\sqrt{\left(\rho+p_{r}\right)^{2}-4 q^{2}} \geq 0$
(SEC)

Beside these energy conditions, a physically reasonable stellar model should also satisfy

E7: $\rho>0, p_{r}>0, p_{t}>0$, and $\rho^{\prime}<0, p_{r}^{\prime}<0$, $p_{t}^{\prime}<0$.

Here, we can see that the validity of the $\mathbf{E} 1$ and $\mathbf{E 2}$ inequalities implies that the $\mathbf{E} \mathbf{4}$ inequality is satisfied. In the same fashion, the validity of the E1, E2 and E7 inequalities ensures that the $\mathbf{E 5}$ and $\mathbf{E 6}$ inequalities are satisfied. So, in general we only need to check the validity of the $\mathbf{E 1}, \mathbf{E 2}, \mathbf{E 3}$ and E7. For our radiating stellar model, it can be seen from the Fig. $4 a-c$ that all these energy conditions are well satisfied throughout the interior of the collapsing star.

We would like to shed some light on the stability of our model by investigating the shear free condition. It has been found that the evolution of the shear depends upon the scalar $Y_{T F}$ which is given by [25]

$Y_{T F}=\Delta+\frac{\rho}{2}-\frac{3 m}{r^{3}}$,

where $m$ is Misner Sharp mass function given by (2.23) or interms of the density and heat flux it can be written as [25]

$m=\frac{1}{2} \int_{0}^{r} R^{2}\left(R^{\prime} \rho+\frac{q \dot{R}}{a(r)} b(r) f(t)\right) d r$.
Here, $R=r b(r) f(t)$ is the radius of the collapsing cloud. Equation (2.59) shows that the scalar $Y_{T F}$ depends upon the density of the cloud $\rho$, pressure anisotropy term $\Delta$ and mass function $m$. It has been shown that for a geodesic fluid, the presence of the inhomogeneous density $\rho$, anisotopy term $\Delta$ and heat flux $q$ can generates the shearing effects within the fluid. To check the stability of the shear free condition for our model, we use the forms of the pressure anisotropy (2.31), density (2.48) and mass function into the Eq. (2.59), which gives $Y_{T F}=\Delta$. This shows that although we have consider the initial shear free collapse, at later time, the presence of the anisotropy term $\Delta$ generates the shearing effects in the fluid distribution.

\section{Thermal properties}

The study of the thermodynamical evolution and the temperature profiles of the radiating stars plays prominent role during dissipative gravitational collapse as they decide the departure from the thermodynamical equilibrium. Previous studies of the shear free and shearing dissipative gravitational collapses shows that the relaxation effects plays significant role in the temperature profiles towards the end state of the dissipative gravitational collapse see [28-30,33,43-45] and references therein. To study the thermodynamical evolution and the temperature profiles of inside the collapsing star, we will use the causal transport equation for the metric (2.1) given by $[27,28,46]$

$$
\begin{aligned}
\tau h_{\mu}^{v} \dot{q}_{\nu}+q_{\mu} & =-k\left(h_{\mu}^{v} \nabla_{\nu} T+T \dot{u}_{\mu}\right) \\
\tau(q b f)_{, t}+q a b f & =-\frac{k(a T)_{, r}}{b f},
\end{aligned}
$$

where, $\alpha>0, \beta>0, \gamma>0$ and $\sigma>0$ are constants and $h^{\mu v}=g^{\mu \nu}+u^{\mu} u^{\nu}$. Also,

$\tau_{c}=\left(\frac{\alpha}{\gamma}\right) T^{-\sigma}, \quad k=\gamma T^{3} \tau_{c}, \quad \tau=\left(\frac{\beta \gamma}{\alpha}\right) \tau_{c}$

are physically reasonable choices of the mean collision time, between massive and massless particles $\tau_{c}$, thermal conductivity $k$ and the relaxation time $\tau$ respectively $[28,35]$. $\tau$ represents the causality index, measures the strength of relaxational effects and $\tau=0$ or $\beta=0$ represents the noncausal case. Using these forms of the physical quantities given in Eq. (3.3), the form of the causal heat transport Eq. (3.2) becomes

$\beta T^{-\sigma}(q b f)_{, t}+q a b f=-\frac{\alpha(a T)_{, r}}{b f} T^{3-\sigma}$.

The noncausal solution of heat equations are obtained by setting $\beta=0$ i.e. $\tau=0$ in the above transport Eq. (3.4) [35] 


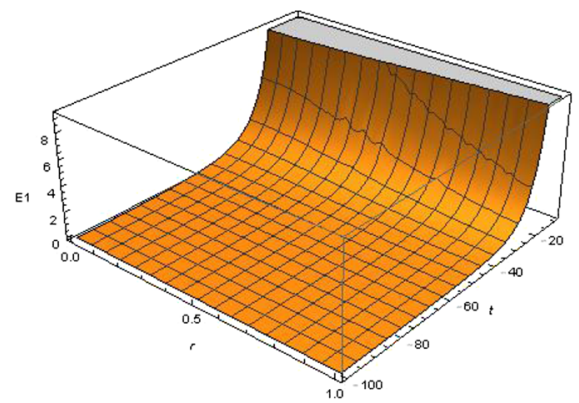

(a)

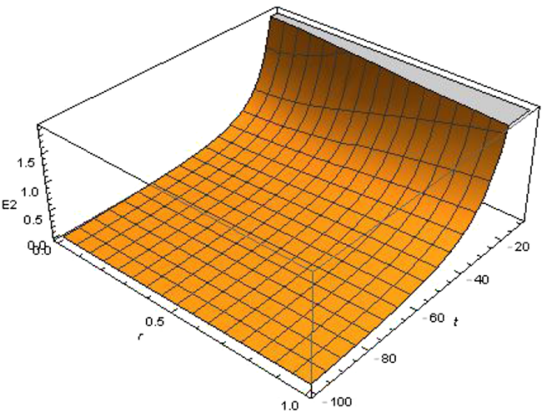

(b)

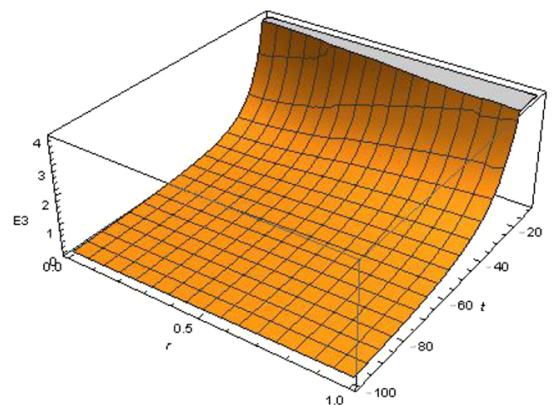

(c)

Fig. 4 a Plot of the energy condition $E 1$ with respect to $t$ and $r$, b plot of the energy condition $E 2$ with respect to $t$ and $r$, $\mathbf{c}$ plot of the energy condition $E 3$ with respect to $t$ and $r$

$$
(a T)^{4}=-\frac{4}{\alpha} \int a^{4} q b^{2} f^{2} d r+F(t), \quad \sigma=0
$$

$\ln (a T)=-\frac{1}{\alpha} \int q b^{2} f^{2} d r+F(t)$.

$\sigma=4$

The causal solution of the above transport Eq. (3.4) are given by [35]

$$
\begin{aligned}
(a T)^{4}=- & \frac{4}{\alpha}\left[\beta \int a^{3} b f(q b f)_{, t} d r+\int a^{4} q b^{2} f^{2} d r\right] \\
& +F(t), \quad \sigma=0
\end{aligned}
$$

$$
\begin{aligned}
(a T)^{4}=- & \frac{4 \beta}{\alpha} \exp \left(-\int \frac{4 q b^{2} f^{2}}{\alpha} d r\right) \\
& \times \int a^{3} b f(q b f)_{, t} d r \exp \\
& \times\left(\int \frac{4 q b^{2} f^{2}}{\alpha} d r\right) \\
+ & F(t) \exp \left(-\int \frac{4 q b^{2} f^{2}}{\alpha} d r\right), \sigma=4
\end{aligned}
$$

where $F(t)$ is the function of integration. The function $F(t)$ is determined by invoking boundary conditions

$$
\left(T^{4}\right)_{\Sigma}=\left(\frac{L_{\infty}}{4 \pi \delta r^{2} b^{2} f^{2}}\right)_{\Sigma} .
$$

where $L_{\infty}$ is the total luminosity for an observer at infinity and $\delta>0$ is constant.

As with previous investigations [32,35], Fig. 5 shows that both the causal and noncausal temperature are same at the boundary of the star. However, at later stages of the collapse, relaxation effects plays significant role and they differ from the noncausal case. This behavior can be seen from the Fig. 5 that with $\beta>0$ the relaxations effects grows and the causal temperature remains greater than that of noncausal temperature throughout the interior of the star. These results are in agreement with the earlier results obtained for the shear free collapse [31,33].

\section{Conclusion}

In this paper, we presented new exact solutions of the Einstein field equations for spherical symmetric systems with heatconducting anisotropic fluid as a collapsing matter. The interior spacetime has been smoothly matched with the exterior Vaidya metric of radiant star across the timelike hypersurface $\Sigma$. For this purpose, we assume the gravitational potentials to be separated into their radial and temporal coordinates. Now to find their exact forms, we consider a special form of the pressure anisotropy as given by the Eq. (2.31) and find one of the metric potential. To find the other gravitational potential, we employed the Karmarkar condition (2.43), which makes the interior spacetime to be class $\mathbf{I}$.

We have investigated the physical quantities like density (2.48), radial pressure (2.49) and tangential pressure (2.50) and it can be seen from the Figs. 1a, b and 2a that they are positive throughout the collapse. From Fig. $2 b$ it is clear that the radial heat flux (2.51) is finite and positive throughout collapse. From Fig. 3a, the negative form of the expansion scalar (2.55) implies the collapsing behavior of the system. We have also investigated the mass function and total luminosity of the collapsing star. It is clear from the Fig. $3 b$ that the mass (2.56) depends linearly on the temporal coordinate. It has been found that the luminosity is time independent for this class of solutions and it radiates uniformly throughout the collapse. Thus the solution is a physically permissible solution of the Einstein theory. We also checked the physically viability of the collapsing model by studying the energy conditions E1, E2 and E3 together with the positivity of the density, radial and tangential pressure profiles and heat flux. It is clear from the Fig. $4 \mathrm{a}-\mathrm{c}$ that all three energy conditions 
Fig. 5 Plot of the causal and noncausal temperature $T$ of the star with respect to $r$ for $\sigma=0$

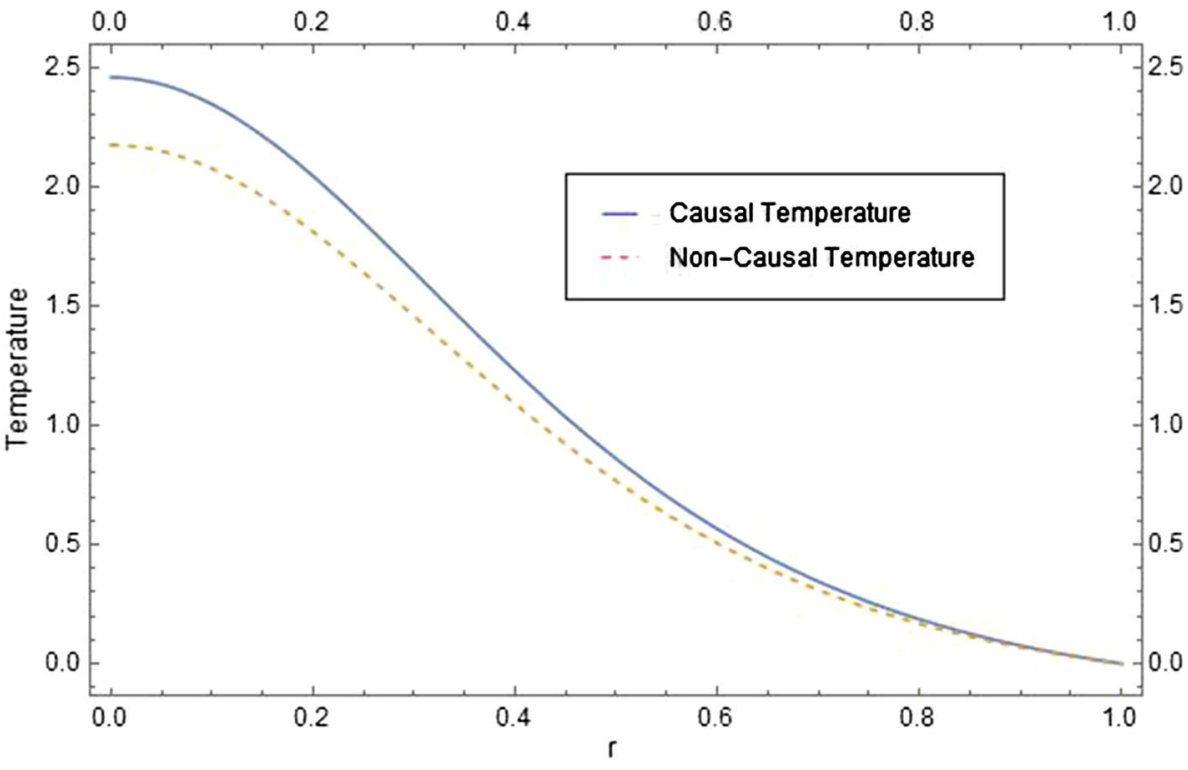

References

E1, E2 and E3 are satisfied. It shows that the present class of solutions seams to be representing the physically viable collapse model.

Also, we have found the surface temperature of the collapsing star at a large past time. It is clear from the Fig. 5 that both the causal and noncausal temperatures are same at the boundary $\Sigma$, however, differs at all interior points of collapsing star. At later stages of the collapse, relaxation effects (3.7) plays significant role and remains greater than that of the noncausal case (3.5). From Fig. 5 shows that with $\beta>0$ the relaxations effects grows and the causal temperature remains greater than that of noncausal temperature throughout the interior of the star. These results are in agreement with the earlier results obtained for the shear free collapse.

Acknowledgements The author thanks Dr. Ayan Chatterjee for several stimulating discussions and continuous encouragement during the course of the work. He also acknowledges his suggestions on various aspects of the work that made the presentation better. The author also acknowledges the comments of the referees which led to several improvements.

Data Availability Statement This manuscript has no associated data or the data will not be deposited. [Authors' comment: This is a theoretical study and does not contain any experimental data.]

Open Access This article is licensed under a Creative Commons Attribution 4.0 International License, which permits use, sharing, adaptation, distribution and reproduction in any medium or format, as long as you give appropriate credit to the original author(s) and the source, provide a link to the Creative Commons licence, and indicate if changes were made. The images or other third party material in this article are included in the article's Creative Commons licence, unless indicated otherwise in a credit line to the material. If material is not included in the article's Creative Commons licence and your intended use is not permitted by statutory regulation or exceeds the permitted use, you will need to obtain permission directly from the copyright holder. To view a copy of this licence, visit http://creativecomm ons.org/licenses/by/4.0/.

Funded by $\mathrm{SCOAP}^{3}$.
1. J.R. Oppenheimer, H. Snyder, Phys. Rev. 56, 455 (1939)

2. S. Datt, Zs. f. Phys. 108, 314 (1938)

3. S.W. Hawking, G.R. Ellis, The Large Scale Structure of Spacetime (Cambridge University Press, Cambridge, 1975)

4. P.S. Joshi, Gravitational Collapse and Spacetime Singularities (Cambridge University Press, Cambridge, 2007)

5. P.S. Joshi, D. Malafarina, Int. J. Mod. Phys. D 20, 2641 (2011)

6. A.K.G. de Oliveira, N.O. Santos, C.A. Kolassis, Mon. Not. R. Astron. Soc. 216, 1001 (1985)

7. C.A. Kolassis, N.O. Santos, D. Tsoubelis, Class. Quant. Grav. 5, 1329 (1988)

8. L. Herrera, G. le Denmat, N.O. Santos, Mon. Not. R. Astron. Soc. 237, 257 (1989)

9. R. Chan MNRAS, 288, 589 (1997)

10. R, Chan, MNRAS, 299, 811 (1998)

11. R. Chan, Astrophys. Sp. Sci. 257, 299 (1998)

12. P.C. Vaidya, Proc. Indian Acad. Sci. A 33, 264 (1951)

13. E.N. Glass, Phys. Lett. A 86, 351 (1981)

14. N.O. Santos, MNRAS 216, 403 (1985)

15. L.P. Eisenhart, Riemannian Geometry (Princeton University Press, Princeton, 1925), p. 97

16. J. Eiesland, Trans. Am. Math. Soc. 27, 213 (1925)

17. K.R. Karmarkar, Proc. Indian Acad. Sci. A 27, 56 (1948)

18. K.N. Singh, N. Pant, Eur. Phys. J. C 76, 524 (2016)

19. S.K. Maurya, Y.K. Gupta, S. Ray, D. Deb, Eur. Phys. J. C 76, 693 (2016)

20. P. Bhar et al., Int. J. Mod. Phys. D 26, 1750078 (2017)

21. N.F. Naidu, M. Govender, S.D. Maharaj, Eur. Phys. J. C 78, 48 (2018)

22. A. Banerjee, S. Chatterjee, N. Dadhich, Mod. Phys. Lett. A 17, 2335 (2002)

23. K. Schwarzschild, Phys.-Math. Klasse, 189 (1916)

24. M. Kohler, K.L. Chao, Z. Naturforsch. Ser. A 20, 1537 (1965)

25. L. Herrera, A. Di Prisco, J. Ospino, Gen. Relativ. Gravit. 42, 1585 (2010)

26. J. Ospino, L.A. Núñez, Eur. Phys. J. C 80, 166 (2020)

27. R. Maartens, Class. Quant. Grav. 12, 1455 (1995)

28. J. Martinez, Phys. Rev. D 53, 6921 (1996)

29. A. Di Prisco, L. Herrera, M. Esculpi, Class. Quant. Grav. 13, 1053 (1996) 
30. L. Herrera, A. Di Prisco, J.L. Hernandez-Pastora, J. Martin, J. Martinez, Class. Quant. Grav. 14, 2239 (1997)

31. L. Herrera, N.O. Santos, Mon. Not. R. Astron. Soc. 287, 161 (1997)

32. M. Govender, S.D. Maharaj, R. Maartens, Class. Quant. Grav. 15, 323 (1998)

33. M. Govender, R. Maartens, S.D. Maharaj, Mon. Not. R. Astron. Soc. 310, 557 (1999)

34. R. Maartens, M. Govender, S.D. Maharaj, Gen. Relativ. Grav. 31, 815 (1999)

35. M. Govender, K. Govinder, Phys. Lett. A 283, 71 (2001)

36. G. Darmois, Mémorial Des Sciences Mathématiques, vol. 25 (Gauthier-Villars, Paris, 1927), p. 1

37. W. Israel, Nuovo Cimento B 44, 1 (1966)

38. W. Israel, Nuovo Cimento B 48, 463 (1966)

39. W.B. Bonnor, P.A. Vickers, Gen. Relativ. Gravit. 13, 29 (1981)
40. C.W. Misner, D.H. Sharp, Phys. Rev. 136, B571 (1964)

41. M.E. Cahill, G.C. McVittie, J. Math. Phys. 11, 1382 (1970)

42. L.P. Eisenhart, Riemannian Geometry (Princeton U. P, Princeton, 1949)

43. L. Herrera, N.O. Santos, Phys. Rev. D 70, 084004 (2004)

44. L. Herrera, G. Le Denmat, N.O. Santos, A. Wang, Int. J. Mod. Phys. D 13, 583 (2004)

45. L. Herrera, Int. J. Mod. Phys. D 15, 2197 (2006)

46. W. Israel and J. Stewart, Ann. Phys.(N.Y.), 118, 341 (1979) 